\title{
The explanation of the Trouton-Noble experiment revisited
}

Saul A. Teukolsky

Citation: American Journal of Physics 64, 1104 (1996); doi: 10.1119/1.18329

View online: https://doi.org/10.1119/1.18329

View Table of Contents: http://aapt.scitation.org/toc/ajp/64/9

Published by the American Association of Physics Teachers

\section{Articles you may be interested in}

On the Trouton-Noble Experiment

American Journal of Physics 36, 936 (1968); 10.1119/1.1974358

On the "explanation" of the null results of Trouton-Noble experiment

American Journal of Physics 61, 428 (1993); 10.1119/1.17236

Question \#26. Electromagnetic field momentum

American Journal of Physics 63, 777 (1995); 10.1119/1.18075

The electromagnetic momentum of static charge-current distributions

American Journal of Physics 82, 869 (2014); 10.1119/1.4879539

Mansuripur's paradox

American Journal of Physics 81, 570 (2013); 10.1119/1.4812445

Examples and comments related to relativity controversies

American Journal of Physics 80, 962 (2012); 10.1119/1.4754867

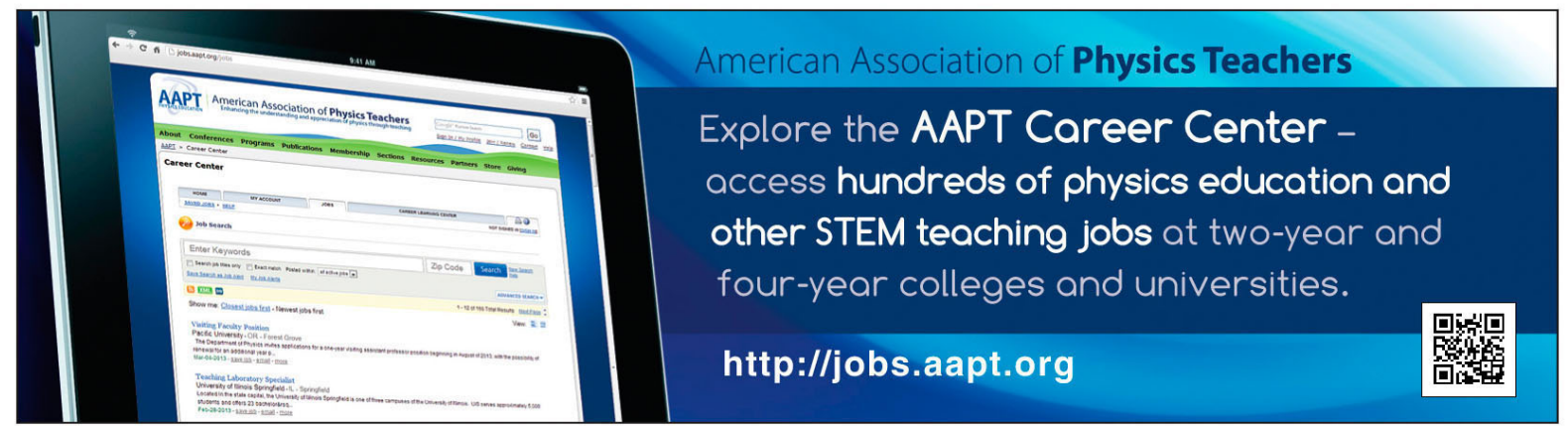




\section{The explanation of the Trouton-Noble experiment revisited}

Saul A. Teukolsky

Departments of Physics and Astronomy, Cornell University, Ithaca, New York 14853

(Received 13 March 1996; accepted 17 April 1996)

One class of explanations of the null result of the Trouton-Noble experiment uses the "standard" definitions of electromagnetic energy and momentum but is quite complicated. Another class is much simpler but appears to require new definitions of electromagnetic energy and momentum. We reconcile the two sets of explanations and show that they are both physically correct. We use the same arguments that have been used in discussions of the famous " $4 / 3$ " factor in the electromagnetic mass of the classical electron. (C) 1996 American Association of Physics Teachers.

\section{THE TROUTON-NOBLE EXPERIMENT}

Shortly before his death in 1901, Fitzgerald proposed an experiment to measure the earth's motion through the ether. ${ }^{1}$ The experiment was subsequently carried out by his former student, Trouton. The idea was to look for the turning motion of a charged parallel plate capacitor suspended at rest in the frame of the earth. Trouton ${ }^{2}$ gave a simple energy argument to predict a torque: The energy of a capacitor at rest in the ether would be

$$
U_{R}=\frac{1}{8 \pi} \int E^{2} d V=\frac{1}{8 \pi} E^{2} V,
$$

where $E$ is the electric field and $V$ is the volume between the plates. When the capacitor is moving through the ether in a direction parallel to the plates, a magnetic field $\mathbf{B}=\mathbf{v} \times \mathbf{E}$ appears. (We use units with the speed of light $c=1$.) Thus the energy for parallel motion is

$$
\begin{aligned}
U_{\|} & =\frac{1}{8 \pi} \int\left(E^{2}+B^{2}\right) d V=\frac{1}{8 \pi}\left(E^{2}+v^{2} E^{2}\right) V \\
& =U_{R}\left(1+v^{2}\right) .
\end{aligned}
$$

When the capacitor is moving perpendicular to the plates, there is no B field, and the energy is

$$
U_{\perp}=U_{R}<U_{\|} \text {. }
$$

Thus there should be a torque tending to rotate the capacitor until the plates are aligned perpendicular to the velocity through the ether, which is the lowest energy state. Since the orientation of the capacitor with respect to the earth's motion through the ether would presumably be different at different times of the day, one could look for this rotational effect. When the experiment was performed by Trouton ${ }^{2}$ and then Trouton and Noble, ${ }^{3}$ they obtained a null result. The experiment was repeated later by Tomaschek ${ }^{4}$ and by Chase. ${ }^{5}$ Chase was prompted by a claim by Miller ${ }^{6}$ to have detected a positive ether-drift velocity in a Michelson-Morley experiment, and by concerns about the reliability of the TroutonNoble and Tomaschek experiments. Chase reported a limit ${ }^{7}$ of $4 \mathrm{~km} / \mathrm{s}$ on any ether-drift velocity, compared with the earth's orbital velocity of $30 \mathrm{~km} / \mathrm{s}$.

\section{NAIVE RELATIVISTIC PREDICTIONS}

Let us see what special relativity predicts if we redo Trouton's argument. Instead of considering motion through the ether, we consider a capacitor moving with respect to some observer with a uniform velocity $\mathbf{v}$. We use bars to denote quantities in the rest frame of the capacitor. If $\mathbf{v}$ is perpendicular to the plates, then it is parallel to the $\mathbf{E}$ field. The Lorentz transformation gives

$$
\mathbf{E}=\overline{\mathbf{E}}, \quad \mathbf{B}=\overline{\mathbf{B}}=0, \quad V=\bar{V} / \gamma,
$$

where $\gamma=\left(1-v^{2}\right)^{-1 / 2}$. Thus the energy is

$$
U_{\perp}=\frac{1}{8 \pi}\left(E^{2}+B^{2}\right) V=\frac{1}{8 \pi} \frac{\bar{E}^{2} \bar{V}}{\gamma}=\frac{1}{\gamma} \bar{U} .
$$

If on the other hand $\mathbf{v}$ is parallel to the plates, and so perpendicular to $\mathbf{E}$, the transformation gives

$$
\mathbf{E}=\gamma \overline{\mathbf{E}}, \quad \mathbf{B}=\gamma(\mathbf{v} \times \overline{\mathbf{E}}), \quad V=\bar{V} / \gamma .
$$

Thus

$$
\begin{aligned}
U_{\|} & =\frac{1}{8 \pi}\left(E^{2}+B^{2}\right) V=\frac{1}{8 \pi}\left(\gamma^{2} \bar{E}^{2}+\gamma^{2} v^{2} \bar{E}^{2}\right) \bar{V} / \gamma \\
& =\gamma\left(1+v^{2}\right) \bar{U} .
\end{aligned}
$$

So $U_{\perp}$ is once again less than $U_{\|}$, and it seems that there should still be an effect. In fact, if we expand the expressions for $U_{\|}$and $U_{\perp}$ to second order in $v$, we see that the predicted effect is twice as big as Trouton's prerelativistic prediction.

However, the relativity principle forbids any such effect. If a capacitor is in equilibrium for an inertial observer at rest with respect to the capacitor, then it cannot appear to be rotating in a uniformly moving frame, for otherwise we could use this effect to single out the preferred frame that has no rotation.

As we shall see, the explanation of the Trouton-Noble experiment requires consideration of the constraint forces holding the capacitor in equilibrium. The error in the naive relativistic treatment in this section is that we ignored the work done by or against these forces. Including the effect of these forces leads to the "correct" transformation law, (5.3)-(5.5), showing that there is no torque.

\section{GENERAL DEFINITION OF 4-MOMENTUM}

Understanding how relativity predicts a null result for the Trouton-Noble experiment involves understanding in detail how electromagnetic energy and momentum should be computed for a moving system. We can easily see that this is not entirely trivial. For example, the electromagnetic field momentum for the case when the capacitor is moving parallel to the plates is 


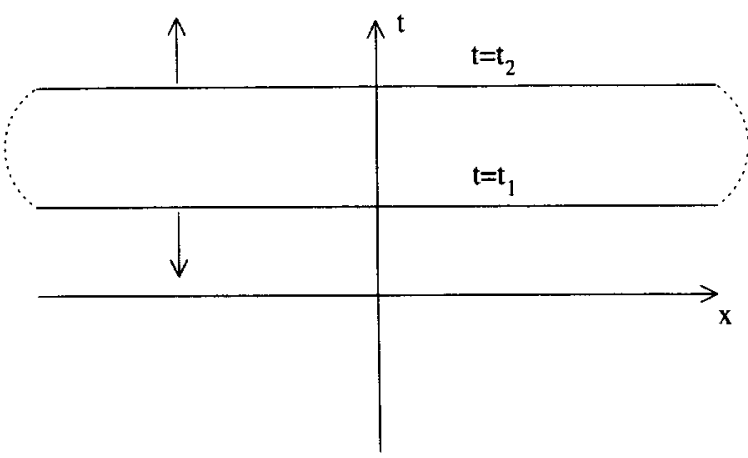

Fig. 1. Region of integration for Eq. (3.6).

$$
\begin{aligned}
|\mathbf{P}| & =\frac{1}{4 \pi}\left|\int \mathbf{E} \times \mathbf{B} d V\right|=\frac{1}{4 \pi} E B V=\frac{1}{4 \pi}(\gamma \bar{E})(\gamma v \bar{E}) \frac{\bar{V}}{\gamma} \\
& =2 \gamma \bar{U} v,
\end{aligned}
$$

which differs by a factor of 2 from what we expect!

To understand this and similar apparent discrepancies, go back to the general definition of the 4-momentum $P^{\alpha}$ of a system in terms of its stress-energy tensor $T^{\alpha \beta}$ :

$$
P^{\alpha}=\int_{\Sigma} T^{\alpha \beta} d^{3} \Sigma_{\beta} .
$$

Here, $d^{3} \Sigma_{\beta}$ is the volume element for any inertial observer, an infinitesimal region of the $t=$ constant hypersurface $\Sigma$ for that observer. (Actually, $\Sigma$ can be any spacelike hypersurface, but we will not need that degree of generality here.) We can write

$$
d^{3} \Sigma_{\beta}=-U_{\beta} d^{3} \sigma,
$$

where $U_{\beta}$, the 4-velocity of the observer, is the unit normal to $\Sigma$. In the observer's rest frame,

$$
U^{\beta}=(1,0,0,0), \quad U_{\beta}=(-1,0,0,0), \quad d^{3} \sigma=d x d y d z .
$$

Local conservation of energy and momentum is expressed by the vanishing divergence of the stress-energy tensor,

$$
T_{, \beta}^{\alpha \beta}=0,
$$

where the comma denotes the partial derivative. This implies the global conservation law, that $P^{\alpha}$ is independent of time: Consider the integral

$$
0=\int_{\Omega} T_{, \beta}^{\alpha \beta} d^{4} \Omega
$$

over the four-dimensional region $\Omega$ bounded by two time slices $t=t_{1}, t=t_{2}$, as shown in Fig. 1 . Close the region $\Omega$ with timelike boundaries at infinity, shown as dotted lines in the figure. By Gauss's theorem, we can convert the integral (3.6) into an integral over the boundary $\Sigma$ of $\Omega$. The "end" regions at infinity do not contribute, since we assume $T^{\alpha \beta} \rightarrow 0$ at infinity. Thus

$$
\begin{aligned}
0 & =\oint_{t=t_{1}, t=t_{2}} T^{\alpha \beta} d^{3} \Sigma_{\beta} \\
& =\int_{t=t_{2}} T^{\alpha \beta} d^{3} \Sigma_{\beta}-\int_{t=t_{1}} T^{\alpha \beta} d^{3} \Sigma_{\beta},
\end{aligned}
$$

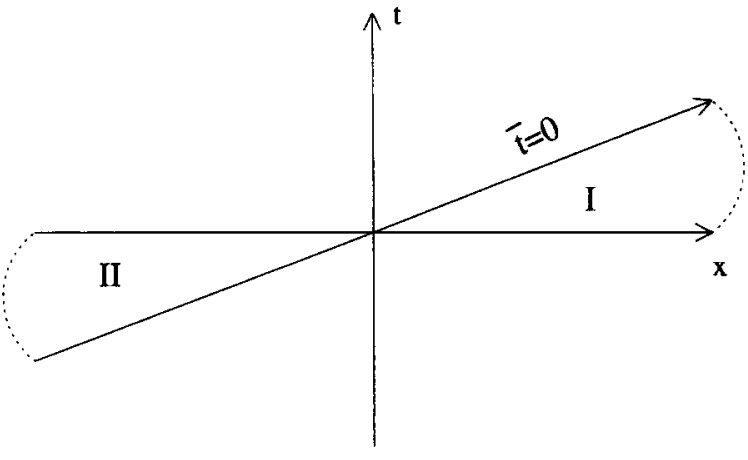

Fig. 2. Region of integration to show that $P^{\alpha}$ is a 4-vector.

where the minus sign appears because the outward normal to $\Sigma$ is in the minus $t$ direction on $t=t_{1}$. Thus

$$
P^{\alpha}\left(t_{2}\right)=P^{\alpha}\left(t_{1}\right) \text {. }
$$

Similarly, we can show that $P^{\alpha}$ defined by (3.2) is, in fact, a 4 -vector. To do this we need to show that $P^{\alpha}$ is independent of the inertial frame used to calculate it. Compare $P^{\alpha}$ computed on the $t=0$ hypersurface of one inertial observer with that computed on the $\bar{t}=0$ hypersurface of a boosted observer, as shown in Fig. 2.

The hypersurfaces intersect forming two four-dimensional regions, I and II. Close each region with timelike boundaries at infinity forming two bounded 4-volumes. Consider the integral (3.6) over volume I, and take into account the signs of the normals as before. We find

$$
\int_{t=0} T^{\alpha \beta} d^{3} \Sigma_{\beta}=\int_{\bar{t}=0} T^{\alpha \beta} d^{3} \Sigma_{\beta} \quad \text { (Region I). }
$$

A similar result holds for region II. Adding the equations for the two regions, we get the desired result:

$$
\int_{t=0} T^{\alpha \beta} d^{3} \Sigma_{\beta}=\int_{t=0} T^{\alpha \beta} d^{3} \Sigma_{\beta} .
$$

\section{THE 4-MOMENTUM OF A COMPOSITE SYSTEM}

Note that in proving that $P^{\alpha}$ is a 4 -vector, it is crucial that $T^{\alpha \beta}{ }_{\beta}=0$. In general, this is true only if $T^{\alpha \beta}$ is the stressenergy tensor for the entire system. If a system is composed of two subsystems, then we will have

$$
T^{\alpha \beta}=T_{(1)}^{\alpha \beta}+T_{(2)}^{\alpha \beta},
$$

but in general neither term on the right-hand side will separately have vanishing divergence. Thus, if we write

$$
P^{\alpha}=P_{(1)}^{\alpha}+P_{(2)}^{\alpha},
$$

where

$$
P_{(1)}^{\alpha}=\int_{\Sigma} T_{(1)}^{\alpha \beta} d^{3} \Sigma_{\beta}, \quad P_{(2)}^{\alpha}=\int_{\Sigma} T_{(2)}^{\alpha \beta} d^{3} \Sigma_{\beta},
$$

then, in general, neither $P_{(1)}^{\alpha}$ nor $P_{(2)}^{\alpha}$ is a 4-vector. However, as long as we use the same $\Sigma$ in the integrals (4.3), then the sum is the total 4-momentum of the system, and it is independent of the choice of $\Sigma$.

As mentioned in Sec. II, to explain the Trouton-Noble experiment, we need to consider the constraint forces hold- 
ing the capacitor in equilibrium. There are forces that keep the two charged plates a fixed distance apart, and also forces that maintain the static charge distribution on the plates despite the electrostatic repulsion within the plane of the plates. For simplicity, we will call these forces the nonelectromagnetic forces, ${ }^{8}$ denoted by a subscript $(n)$, to distinguish them from the electromagnetic forces, denoted by a subscript $(e)$. Thus we write

$$
P^{\alpha}=P_{(e)}^{\alpha}+P_{(n)}^{\alpha},
$$

where

$$
P_{(e)}^{\alpha}=\int_{\Sigma} T_{(e)}^{\alpha \beta} d^{3} \Sigma_{\beta}, \quad P_{(n)}^{\alpha}=\int_{\Sigma} T_{(n)}^{\alpha \beta} d^{3} \Sigma_{\beta} .
$$

In fact, it is not even necessary that the constraint forces be derivable from a stress-energy tensor-in a macroscopic description they might simply be described by a force density $f_{(n)}^{\alpha}$. In this case, the local conservation law (3.5) is replaced by

$$
T_{(e), \beta}^{\alpha \beta}+f_{(n)}^{\alpha}=0 .
$$

Since

$$
T_{(e), \beta}^{\alpha \beta}=-F^{\alpha \beta} J_{\beta} \equiv f_{(e)}^{\alpha},
$$

where $F^{\alpha \beta}$ is the electromagnetic field tensor and $J_{\beta}$ the 4-current density, we have

$$
f_{(n)}^{\alpha}=-f_{(e)}^{\alpha}=-(\mathbf{E} \cdot \mathbf{J}, \rho \mathbf{E}+\mathbf{J} \times \mathbf{B}) .
$$

In the second line we have written the components in any inertial frame in terms of the Joule heating and the Lorentz force density.

Now, for a macroscopic nonelectromagnetic force, one defines the "4-momentum" on a hypersurface $\Sigma$ by

$$
P_{(n)}^{\alpha}=\int f_{(n)}^{\alpha} d^{4} \Omega
$$

where the integral extends from $t=-\infty$ up to the chosen hypersurface $\Sigma$, and we assume $f_{(n)}^{\alpha} \rightarrow 0$ as $t \rightarrow-\infty$. This definition is explained in Appendix A. There it is also shown that the definition (4.9) reduces to the definition (4.5) in the case that $f_{(n)}^{\alpha}$ is, in fact, the divergence of a stress-energy tensor. As pointed out earlier, $P_{(n)}^{\alpha}$ is not in general a 4-vector.

\section{EXPLANATIONS OF THE TROUTON-NOBLE EXPERIMENT}

We are finally ready to address the explanation of the null result of the Trouton-Noble experiment. Historically, there have been two classes of explanations. The most frequent set of explanations ${ }^{9-13}$ is equivalent to having any inertial observer make the choice $t=$ constant for the hypersurface $\Sigma$ in (4.5). This choice has the advantage that $P_{(e)}^{\alpha}$ takes on its familiar form in the observer's frame:

$$
P_{(e)}^{\alpha}=\int T^{\alpha 0} d^{3} x
$$

that is,

$$
\begin{aligned}
& P_{(e)}^{0}=\frac{1}{8 \pi} \int\left(E^{2}+B^{2}\right) d^{3} x=\int u d^{3} x, \\
& \mathbf{P}_{(e)}=\frac{1}{4 \pi} \int(\mathbf{E} \times \mathbf{B}) d^{3} x=\int \mathbf{g} d^{3} x .
\end{aligned}
$$

However, as we have seen above, $P_{(e)}^{\alpha}$ does not transform like a 4-vector if $f_{(e)}^{\alpha} \neq 0$. The "explanations" of the null result of the Trouton-Noble experiment are equivalent to determining $P_{(n)}^{\alpha}$ and then showing that the sum $P^{\alpha}=P_{(e)}^{\alpha}+P_{(n)}^{\alpha}$ behaves as expected for a 4-vector; i.e., if in the rest frame

$$
P^{\bar{\alpha}}=(\bar{U}, \mathbf{0}),
$$

then in the observer's frame

$$
P^{\alpha}=(\gamma \bar{U}, \gamma \bar{U} \mathbf{v}) .
$$

The time component of Eq. (5.4) shows, in particular, that

$$
U=\gamma \bar{U},
$$

independent of the orientation of the system. Thus there is no torque on the moving capacitor.

The explanations in this class require a certain degree of ingenuity and perseverance to track down and calculate all the nonelectromagnetic contributions. Readers can judge for themselves the accuracy of this assessment by consulting, e.g., Refs. 11 and 12 .

The second class of explanations ${ }^{14-16}$ is equivalent to the choice $\bar{t}=$ constant in the rest frame of the system for the hypersurface $\Sigma$ in (4.5). All observers agree to use this choice of $\Sigma$. This choice has the disadvantage that it leads to unfamiliar expressions for $P_{(e)}^{\alpha}$ in the observer's frame:

$$
P_{(e)}^{\alpha}=\int_{\bar{t}=\text { const }} T_{(e)}^{\alpha \beta} d^{3} \Sigma_{\beta}=-\int T_{(e)}^{\alpha \beta} U_{\beta} d^{3} \bar{x} .
$$

Since $d^{3} \bar{x}=\gamma d^{3} x$ and $U_{\beta}=(-\gamma, \gamma \mathbf{v})$, we get

$$
\begin{aligned}
& P_{(e)}^{0}=\gamma^{2} \int\left(T^{00}-T^{0 i} v_{i}\right) d^{3} x, \\
& P_{(e)}^{i}=\gamma^{2} \int\left(T^{i 0}-T^{i j} v_{j}\right) d^{3} x,
\end{aligned}
$$

or

$$
\begin{aligned}
& P_{(e)}^{0}=\gamma^{2} \int(u-\mathbf{v} \cdot \mathbf{g}) d^{3} x, \\
& \mathbf{P}_{(e)}=\gamma^{2} \int(\mathbf{g}-\mathbf{v} \cdot \mathbf{T}) d^{3} x,
\end{aligned}
$$

where $\mathbf{T}$ is the Maxwell stress tensor. Here, all quantities have been expressed in the observer's frame.

The key advantage of this choice is that it leads to considerable simplification for a system that has no momentum density $\overline{\mathbf{g}}$ in the rest frame, for example, an electromagnetic system with $\overline{\mathbf{B}}=0$. In the rest frame, $\mathbf{v}=0$ and the above expressions reduce to

$$
P_{(e)}^{\overline{0}}=\int \bar{u} d^{3} \bar{x}, \quad \overline{\mathbf{P}}_{(e)}=0 .
$$

In the observer's frame, since $\overline{\mathbf{B}}=0$, Eqs. (2.1) and (2.3) give 


$$
\mathbf{B}=\mathbf{v} \times \mathbf{E} .
$$

Thus

$$
\mathbf{v} \cdot(\mathbf{E} \times \mathbf{B})=(\mathbf{v} \times \mathbf{E}) \cdot \mathbf{B}=B^{2} .
$$

Hence, Eq. (5.8) becomes

$$
P_{(e)}^{0}=\gamma^{2} \int\left(\frac{E^{2}+B^{2}}{8 \pi}-\frac{B^{2}}{4 \pi}\right) d^{3} x=\frac{\gamma^{2}}{8 \pi} \int\left(E^{2}-B^{2}\right) d^{3} x .
$$

Since $E^{2}-B^{2}$ is a Lorentz invariant, $E^{2}-B^{2}=\bar{E}^{2}-\bar{B}^{2}=\bar{E}^{2}$, and so

$$
P_{(e)}^{0}=\frac{\gamma}{8 \pi} \int \bar{E}^{2} d^{3} \bar{x}=\gamma \bar{U} .
$$

Similarly,

$$
\begin{aligned}
4 \pi \mathbf{v} \cdot \mathbf{T} & =\mathbf{v} \cdot\left[-\mathbf{E E}-\mathbf{B B}+\frac{1}{2}\left(E^{2}+B^{2}\right) \mathbf{1}\right] \\
& =-(\mathbf{v} \cdot \mathbf{E}) \mathbf{E}+\frac{1}{2}\left(E^{2}+B^{2}\right) \mathbf{v},
\end{aligned}
$$

since $\mathbf{v} \cdot \mathbf{B}=0$. Also,

$$
4 \pi \mathbf{g}=\mathbf{E} \times \mathbf{B}=\mathbf{E} \times(\mathbf{v} \times \mathbf{E})=E^{2} \mathbf{v}-(\mathbf{E} \cdot \mathbf{v}) \mathbf{E} .
$$

Substituting Eqs. (5.15) and (5.16) in (5.9), we get

$$
\mathbf{P}_{(e)}=\frac{\gamma^{2} \mathbf{v}}{8 \pi} \int\left(E^{2}-B^{2}\right) d^{3} x=\gamma \bar{U} \mathbf{v}
$$

Thus the quantity $P_{(e)}^{\alpha}$ does transform like a 4-vector when it is computed with this prescription for a system with $\overline{\mathbf{B}}=0$. This makes the description of the behavior of the system much simpler than with the "usual" choice of $\Sigma$ for computing the 4-momentum of a composite system. For example, we know immediately from the fact that $P_{(e)}^{\bar{\alpha}}$ has the form of Eq. (5.3) that the energy (5.5) has no directional dependence and so there is no torque associated with this 4-momentum.

What about the nonelectromagnetic forces in this description? Clearly, since the sum (4.4) is a 4-vector, it must be true that $P_{(n)}^{\alpha}$ is also a 4-vector in this description. In Appendix $B$ we show that it is the trivial 4-vector

$$
P_{(n)}^{\alpha}=0 \text {, }
$$

and hence also has no associated torque.

\section{DISCUSSION}

We have approached the explanation of the TroutonNoble experiment by considering the energy of the moving capacitor. One can also consider the effect of the various forces in the system (see, e.g., Ref. 11). One gets apparent paradoxes because the direction of a force can change under a Lorentz transformation. One must in fact be careful whether one is discussing forces or force densities. A force density is the divergence of a stress-energy tensor and so is a 4-vector. Individual force densities can be correctly transformed with a Lorentz transformation. Moreover, if the resultant force density cancels in one frame, it cancels in all frames. The condition for equilibrium of a body is that the resultant force vanishes, which requires integrating the force density over the volume of the body at some instant. If instead one splits the total force into forces due to subsystems, each of which is an integral over the volume [cf. Eq. (A2)], then these constituent forces are not in general 4-vectors. Using the Lorentz transformation law blindly for these constituent forces can lead to paradoxes. However, provided one chooses the same $t=$ constant hypersurface for all the volume integrals, the total force is well defined and one can show that equilibrium in the co-moving frame corresponds to equilibrium in a moving frame.

As we have seen, reconciling the different energy-based explanations of the Trouton-Noble experiment involves understanding how the 4-momentum of composite systems transforms. The noncovariance of the electromagnetic 4-momentum when sources are present is well known, but not often emphasized in introductory treatments. For radiation fields, there are no sources and hence no problems. In general, however, one must consider the nonelectromagnetic contributions to recover a true 4-vector quantity. The split into electromagnetic and nonelectromagnetic parts is quite arbitrary and neither piece separately is a 4-vector in general. However, with the decomposition made by using the rest frame of the system as in Eqs. (5.8) and (5.9), for a large class of systems the electromagnetic and nonelectromagnetic pieces are separately covariant. The class of such systems is that for which there is no net electromagnetic momentum in the rest frame:

$$
\int \overline{\mathbf{g}} d^{3} \bar{x}=0
$$

It includes in particular systems with $\overline{\mathbf{B}}=0$.

Note that for the simpler class of explanations of the Trouton-Noble experiment, in fact no new definitions of electromagnetic energy and momentum are required. These quantities are still integrals of the corresponding densities over all space. However, by integrating these densities in the rest frame of the system rather than in the observer's frame, one deals with quantities that are true 4-vectors. It is thus not surprising that the resulting description is simpler.

The splitting of the 4-momentum into electromagnetic and nonelectromagnetic contributions has been extensively discussed ${ }^{16-18}$ in connection with the famous " $4 / 3$ " factor in the electromagnetic mass of the classical electron. The viewpoint presented in this paper follows that of Rohrlich ${ }^{16}$ in his discussion of that problem. In fact, the arguments in the body of this paper are simply an extension of Rohrlich's arguments from the classical electron mass to the Trouton-Noble experiment.

Proponents of each class of explanations described in Sec. $\mathrm{V}$ have had a tendency to label the other class of explanations as misguided or wrong. For example, we find in Ref. 15, "An explanation of this paradox was invented by Laue and repeated by Pauli and others." Or in Ref. 11, "Contrary to what was claimed earlier, a 'satisfactory' explanation of the null results of the Trouton-Noble experiment in no way demands a change in the standard definition of the electromagnetic energy-momentum density." I believe this kind of statement simply obscures the main point: There is no physical content to insisting on a splitting of 4-momentum into pieces that are not 4-vectors. With any given splitting, one gets a perfectly valid explanation provided one accounts for all the contributions to the 4-momentum. It is then a matter of taste which particular explanation is most satisfactory. My own taste is for simplicity, in which case the splitting that uses the rest frame of the system is the "best" explanation of the Trouton-Noble experiment. 


\section{ACKNOWLEDGMENTS}

I thank Kurt Gottfried for interesting me in this problem, and thank him and David Mermin for helpful discussions. This work was supported, in part, by the National Science Foundation.

\section{APPENDIX A}

Here, we amplify the definition (4.9) of 4-momentum in terms of force density. Consider first calculating 3-momentum from force:

$$
\mathbf{P}=\int_{-\infty}^{t} \frac{d \mathbf{P}}{d t} d t=\int_{-\infty}^{t} \mathbf{F} d t .
$$

Here, we assume that $\mathbf{F} \rightarrow 0$ and $\mathbf{P} \rightarrow 0$ as $t \rightarrow-\infty$, so that the momentum at any instant is just the accumulated result of the force. Now, if the force is expressed in terms of a force density,

$$
\mathbf{F}=\int \mathbf{f} d^{3} x
$$

we get

$$
\mathbf{P}=\int_{-\infty}^{t} d t \int d^{3} x \mathbf{f}
$$

which is just the spatial component of Eq. (4.9). The time component of Eq. (4.9) is simply the statement that the energy is the accumulated rate of doing work on the system, and the rate of doing work follows from the relation

$$
f^{0}=\mathbf{f} \cdot \mathbf{v}
$$

between the components of the 4-force density.

In practice, we typically end up considering the difference of $P_{(n)}^{\alpha}$ in Eq. (4.9) on two different hypersurfaces $\Sigma_{1}$ and $\Sigma_{2}$, in which case the integral (4.9) will extend over a 4-volume $\Omega$ that is finite in $t$. In a system like that of the Trouton-Noble experiment, we can instead imagine "clamping"' the charges simultaneously in the rest frame at $\bar{t}_{0}$, when they have been assembled into their final equilibrium positions. Then the force density vanishes for $\bar{t}<\bar{t}_{0}$, and again the 4-volume is finite in time.

Note that if $f_{(n)}^{\alpha}=T_{(n), \beta}^{\alpha \beta}$, then Eq. (4.9) gives

$$
P_{(n)}^{\alpha}=\int T_{(n), \beta}^{\alpha \beta} d^{4} \Omega=\int T_{(n)}^{\alpha \beta} d^{3} \Sigma_{\beta} .
$$

In other words, the definition (4.9) reduces to the definition (4.5) in the case that $f_{(n)}^{\alpha}$ is, in fact, the divergence of a stress-energy tensor.

\section{APPENDIX B}

Here, we verify Eq. (5.18). Consider, first, the force holding the plates apart. In the rest frame, Eq. (4.8) gives

$$
\begin{aligned}
& f^{\overline{0}}=0, \\
& \overline{\mathbf{f}}=\bar{\rho} \overline{\mathbf{E}}=\bar{\sigma} \theta\left(\bar{t}-\bar{t}_{0}\right)[\delta(\bar{x})-\delta(\bar{x}-d)] \overline{\mathbf{E}} .
\end{aligned}
$$

Here, $\bar{\sigma}$ is the surface charge density on the plates, taken to be the planes $\bar{x}=0$ and $\bar{x}=d$, and $\theta$ is a Heaviside step function to denote that the force density vanishes for $\bar{t}<\bar{t}_{0}$, as discussed in Appendix A. Thus

$$
P_{(n)}^{0}=\int d^{4} x f^{0}=\int d^{4} \bar{x} \gamma\left(f^{\overline{0}}-\mathbf{v} \cdot \overline{\mathbf{f}}\right)=0 .
$$

Here, we have used the invariance $d^{4} x=d^{4} \bar{x}$ and the fact that the two terms in Eq. (B2) give equal and opposite contributions.

Similarly,

$$
\overline{\mathbf{P}}_{(n)}=\int d^{4} x \mathbf{f}=\int d^{4} \bar{x}\left(\gamma \overline{\mathbf{f}}_{\|}+\overline{\mathbf{f}}_{\perp}-\gamma \mathbf{v} f^{\overline{0}}\right)=0
$$

for the same reasons as $P_{(n)}^{0}=0$. [In Eq. (B4), the $\|$ and $\perp$ components are with respect to the direction of $\mathbf{v}$.]

The repulsive forces in the plane of each capacitor plate also contribute no net 4-momentum. The reason is again symmetry: In the rest frame, choose the origin of the coordinates in the center of one of the plates, which we take to lie in the $\overline{y z}$-plane. Then the $\bar{y}$ component of the repulsive force on a charge element at $(\bar{y}, \bar{z})$ is in the direction of increasing $\bar{y}$ for $\bar{y}>0$, and decreasing $\bar{y}$ for $\bar{y}<0$. This is similar for the $\bar{z}$ component of the force. Thus, when we integrate over $d \bar{y} d \bar{z}$ in Eqs. (B.3) and (B.4), we get zero by symmetry.

Note: See also Robert H. Romer, "Question \#26...," Am. J. Phys. 63 (9), 777-779 (1995), and three "Answers" by McDonald, Rohrlich, and Holstein in the January, 1996 issue.

${ }^{1}$ For an account of this history, see E. T. Whitaker, A History of the Theories of Aether and Electricity (Thomas Nelson, London, 1953), Vol. II, pp. 28 and 29.

${ }^{2} \mathrm{~F}$. T. Trouton, "The results of an electrical experiment, involving the relative motion of the earth and ether, suggested by the late Professor Fitz Gerald," Sci. Trans. R. Dublin Soc. 7, 379-384 (1902).

${ }^{3} F$. T. Trouton and H. R. Noble, "The mechanical forces acting on a charged electric condenser moving through space," Philos. Trans. R. Soc. London Ser. A 202, 165-181 (1904); abstract in Proc. R. Soc. London 72, 132-133 (1903).

${ }^{4} \mathrm{R}$. Tomaschek, "Über Versuche zur Auffindung elektrodynamischer Wirkungen der Erdbewegung in grossen Höhen I," Ann. Phys. 78, 743756 (1926); 80, 509-514 (1926).

${ }^{5} \mathrm{C}$. T. Chase, "A repetition of the Trouton-Noble ether drift experiment," Phys. Rev. 28, 378-383 (1926).

${ }^{6}$ D. C. Miller, "Significance of the ether-drift experiments of 1925 at Mount Wilson," Science 63, 433-443 (1926).

${ }^{7}$ Chase was uncertain whether the "theoretical" prediction for the torque should use the vacuum value of the capacitance in computing the electrostatic energy (1.1), or the actual value, which is larger by the magnitude of the dielectric constant of the material between the plates. It seems clear from Trouton's argument that it is the actual energy that is involved, not the vacuum energy, in which case Chase's limit is about $1 \mathrm{~km} / \mathrm{s}$. Miller's reported ether-drift velocity was about $10 \mathrm{~km} / \mathrm{s}$.

${ }^{8}$ While from a microscopic point of view, the origin of the constraint forces might be electromagnetic, the point of the notation is to distinguish the "original" electromagnetic quantities we started analyzing in the capacitor system from the constraint quantities that we subsequently realize must be present.

${ }^{9}$ M. v. Laue, "Zur dynamik der relativitätstheorie," Ann. Phys. 35, 524542 (1911).

${ }^{10} \mathrm{~W}$. Pauli, Theory of Relativity (Pergamon, New York, 1958), pp. 127-130.

${ }^{11}$ A. K. Singal, "On the 'explanation' of the null results of the TroutonNoble experiment," Am. J. Phys. 61, 428-433 (1993).

${ }^{12}$ A. K. Singal, "Energy-momentum of the self-fields of a moving charge in classical electromagnetism," J. Phys. A 25, 1605-1620 (1992)

${ }^{13}$ A. P. Lightman, W. H. Press, R. H. Price, and S. A. Teukolsky, Problem Book in Relativity and Gravitation (Princeton U.P., Princeton, 1975), Problem 5.8.

${ }^{14}$ J. W. Butler, "On the Trouton-Noble experiment," Am. J. Phys. 36, 936-941 (1968). 
${ }^{15} \mathrm{~J}$. W. Butler, “A proposed electromagnetic momentum-energy 4-vector for charged bodies," Am. J. Phys. 37, 1258-1272 (1969).

${ }^{16} \mathrm{~F}$. Rohrlich, "Comment on the preceding paper by T. H. Boyer," Phys. Rev. D 25, 3251-3255 (1982).
${ }^{17} \mathrm{~T}$. H. Boyer, "Classical model of the electron and the definition of electromagnetic field momentum,' Phys. Rev. D 25, 3246-3250 (1982).

${ }^{18} \mathrm{~F}$. Rohrlich, "Self-energy and stability of the classical electron," Am. J. Phys. 28, 639-643 (1960).

\title{
Teaching physics with 670-nm diode lasers-experiments with Fabry-Perot cavities
}

\author{
R. A. Boyd, J. L. Bliss, and K. G. Libbrecht ${ }^{\mathrm{a})}$ \\ Norman Bridge Laboratory of Physics, California Institute of Technology 12-33, Pasadena, \\ California 91125
}

(Received 19 February 1996; accepted 9 May 1996)

\begin{abstract}
In a previous paper we described details of the construction of stabilized $670-\mathrm{nm}$ diode lasers for use in undergraduate physics laboratories. We report here a series of experiments that can be performed using the 670-nm diode laser, a homemade scanning Fabry-Perot cavity, a helium-neon laser, a simple photodiode, and a few pieces of electronics hardware. The experiments include: (1) an introduction to the scanning confocal Fabry-Perot cavity, and to its use as an optical spectrum analyzer; (2) laser frequency modulation and observation of FM sidebands using the optical spectrum analyzer; and (3) the Pound-Drever method for servo-locking a Fabry-Perot cavity to a laser. These experiments are relatively easy to set up and perform, yet they demonstrate a number of useful optical principles and experimental techniques. (C) 1996 American Association of Physics Teachers.
\end{abstract}

\section{INTRODUCTION}

In a previous paper by Libbrecht et $_{\text {al. }}{ }^{1}$ we described the construction of stabilized $670-\mathrm{nm}$ semiconductor diode lasers for use in undergraduate teaching laboratories. These inexpensive visible lasers emit tunable coherent light which can be used to perform a number of interesting and fundamental physics experiments. The lasers provide the foundation for a new 9-week (one quarter) senior physics lab course at Caltech, which consists of a series of experiments in optical and atomic physics.

An attractive feature of the Caltech course is that it is track based, i.e., students all follow the same track in parallel. The course begins with simpler experiments to build up experience with the equipment and the physics; students then move on to more complex experiments as the course progresses. The equipment needed for these experiments is sufficiently inexpensive that several setups can operate simultaneously, which is necessary for a track-based course. ${ }^{2}$

We describe here a series of three experiments involving lasers and Fabry-Perot cavities. The first (and simplest) experiment consists of aligning two spherical mirrors to form a confocal cavity, and using the cavity as an optical spectrum analyzer. This familiarizes the students with basic FabryPerot cavity concepts and gives them experience aligning an optical cavity.

In the second experiment, the students use their optical spectrum analyzer to observe FM sidebands on a diode laser beam. The sidebands are produced by if modulation of the diode's injection current. The shape of the FM sidebands is readily calculated, and students have the opportunity to compare their calculated spectra with observed spectra.

In the third experiment, the students use the Pound-
Drever method to lock a Fabry-Perot resonance frequency to the diode laser's frequency. FM sidebands are added to the optical carrier, and an optical rf circuit produces an electronic error signal which is related to the difference between the laser frequency and the resonance frequency of the nearest longitudinal cavity mode. The error signal is then used to servo-lock the cavity to the laser. In our experience this experiment is particularly popular. It involves concepts that are both powerful and fairly easy to grasp, and most of our undergraduate students (physics majors) are unfamiliar with if technology and servo mechanisms at the beginning of the course.

The first of these three experiments is not unlike other undergraduate laboratory experiments involving FabryPerot cavities. ${ }^{3-6}$ The other two experiments, however, since they require the addition of rf sidebands to the laser, are usually considered too expensive for a teaching lab. This is no longer the case, since sidebands can be easily added to a diode laser's output via rf modulation of the laser's injection current, as is described below.

\section{THE OPTICAL SPECTRUM ANALYZER}

Fabry-Perot cavities are in widespread use in optical physics, for such applications as sensitive wavelength discriminators and for building up large light intensities from modest input powers. In this first experiment students assemble a Fabry-Perot cavity and examine its properties. Figure 1 shows the basic Fabry-Perot cavity, consisting of two spherical mirrors separated by a distance $L$. An excellent detailed discussion of the properties of Fabry-Perot cavities is given by Yariv. ${ }^{7}$ 